\title{
INADMISSIBLE RECURSION THEORY
}

\author{
BY S. D. FRIEDMAN AND G. E. SACKS ${ }^{1}$ \\ Communicated by Solomon Feferman, September 23, 1976
}

Let $\beta$ be a limit ordinal, and let $S_{\beta}$ be stage $\beta$ of Jensen's $S$ hierachy for $L$ (cf. $\left[1\right.$, p. 82]). $S_{\beta}$ is the setting for $\beta$-recursion theory, an extension of recursion theory on $\Sigma_{1}$ admissible, initial segments of $L$ (so-called $\alpha$-recursion theory) to rudimentarily closed, initial segments of $L$. A set $A \subset S_{\beta}$ is said to be $\beta$-recursively enumerable ( $\beta$-r.e.) if it is $\Sigma_{1}$ definable over $S_{\beta}$. If $\beta$ is $\Sigma_{1}$ admissible, i.e. $S_{\beta}$ satisfies $\Sigma_{1}$ replacement, then many theorems about ordinary r.e. sets $(\beta=\omega)$ remain true when r.e. is replaced by $\beta$-r.e. (cf. [3], [5] and [6]). The results below, particularly the solution to Post's problem devised by Friedman in Theorem 3, suggest that the assumption of $\Sigma_{1}$ admissibility is superfluous. This outcome is in the natural order of events, because arguments in the $\Sigma_{1}$ admissible case often consist of showing that the use of $\Sigma_{2}$ or $\Sigma_{3}$ admissibility when $\beta=\omega$ was unnecessary. It is a desirable outcome in that it implies that the methods of ordinary recursion theory can be applied to all levels of the $J$ hierarchy for $L$ (cf. [1]).

The definitions in this paragraph are drawn from $\alpha$-recursion theory. $A$ is $\beta$-recursive ( $\beta$-rec.) if $A$ and $S_{\beta}-A$ are $\beta$-r.e. A function $f$ is $\beta$-rec. if its graph is. $x$ is $\beta$-finite if $x \in S_{\beta}$. Let $F(e, x)$ be a $\Sigma_{1}$ formula such that $\left\{F(e, x) \mid e \in S_{\beta}\right\}$ is a list of all $\Sigma_{1}$ formulas with free variable $x$ and parameters in $S_{\beta}$. For any $A \subset S_{\beta},\{e\}^{A}(x)$ has $y$ as a value if $S_{\beta}$ satisfies

$$
(E u)(E v)\left[u \subset A \& v \subset S_{\beta}-A \& F(e,\langle x, y, u, v\rangle)\right] \text {. }
$$

$f$ is weakly $\beta$-recursive in $A\left(f \leqslant_{w \beta} A\right)$ if for some $e$ and all $x,\{e\}^{A}(x)=f(x)$. $B \leqslant_{w \beta} A$ if its characteristic function $C_{B} \leqslant_{w \beta} A$. Let $B^{*}$ be the set of all $\beta$ finite $x \subset B . B$ is $\beta$-recursive in $A\left(B \leqslant_{\beta} A\right)$ if $B^{*} \leqslant_{w \beta} A$ and $\left(S_{\beta}-B\right)^{*} \leqslant_{w \beta} A$.

Suppose $A$ is nonempty and $\beta$-r.e. In the admissible case an enumeration of $A$ is a $\beta$-rec. map $f$ of $\beta$ onto $A$. This definition is unsuitable in the inadmissible case, because there may be a $\delta<\beta$ such that $f[\delta]$, the range of $f$ restricted to $\delta$, is unbounded in $S_{\beta}$. A notion of enumeration which suits both cases is as follows. Let $G(x, y)$ be a $\Delta_{0}$ formula such that $x \in A$ iff $S_{\beta} \vDash(E y) G(x, y)$. Let $A^{\delta}$ be the set of all $x \in S_{\delta}$ such that $S_{\delta} \vDash(E y) G(x, y)$. Then $\left\{A^{\delta} \mid \delta<\beta\right\}$ is an enumeration of $A$, namely a nondecreasing, $\beta$-recursive sequence (of length $\beta$ ) of $\beta$-finite sets whose union is $A$.

AMS (MOS) subject classifications (1970). Primary 02 F27.

${ }^{1}$ The second author was supported in part by NSF Grant MCS 76-10430. 
$A$ is said to be tamely $\beta$-r.e. (t.r.e.) if there is an enumeration $\left\{A^{\delta}\right\}$ of $A$ such that $(x)(E \delta)\left[x \in S_{\beta} \& x \subset A \rightarrow x \subset A^{\delta}\right]$. T.r.e. sets are amenable to some of the arguments of admissible recursion theory. Unfortunately there are many $\beta$ 's such that every t.r.e. set is $\beta$-recursive in 0 , the empty set.

Theorem 1. Assume $\beta$ is not $\Sigma_{1}$ admissible. Let $C$ be the complete $\beta$-r.e. set. Then there exists a $\beta$-rec. A such that $0<_{\beta} A<_{\beta} C, C \leqslant_{w \beta} A$, and every set which is either $\beta$-rec. or t.r.e. is $\beta$-rec. in $A$.

The $\Sigma_{1}$ cofinality of $\beta(\sigma 1 \operatorname{cf}(\beta))$ is the least $\gamma$ such that $\beta=\bigcup f[\gamma]$ for some $\beta$-rec. $f$. The $\Sigma_{1}$ projectum of $\beta(\sigma 1 p(\beta))$ is the least $\gamma$ such that some oneone $\beta$-rec. $f$ maps $\beta$ into $\gamma . A$ is regular if $A \cap x \in S_{\beta}$ for all $x \in S_{\beta}$. $\gamma$ is $\beta$ recursively regular if there is no $\beta$-rec. $f$ such that $\gamma=\bigcup f[\delta]$ for some $\delta<\gamma$.

TheOREM 2. Assume $\sigma 1 c f(\beta) \geqslant \sigma 1 p(\beta)$. Then there exist two regular, tamely $\beta$-r.e. sets such that neither is weakly $\beta$-recursive in the other.

THEOREM 3 (S. Friedman [2]). Assume $\sigma 1 p(\beta)$ is $\beta$-recursively regular. Then there exist two $\beta$-r.e. subsets of $\sigma 1 p(\beta)$ such that neither is weakly $\beta$-recursive in the other.

The proof of Theorem 2 is closely tied to ideas associated with admissible recursion theory. Its converse has been proved by W. Maass [4]. The proof of Theorem 3 has several features with no antecedents in the admissible case, the most notable being the use of a $\beta$-recursive version of Jensen's $\diamond$ principle [1, p. 48] to overcome the fact that the $\beta$-r.e. sets constructed cannot be required to be tame.

\section{REFERENCES}

1. K. J. Devlin, Aspects of constructibility, Lecture Notes in Math., vol. 354, SpringerVerlag, Berlin and New York, 1973. MR 51 \#12527.

2. S. D. Friedman, Recursion on inadmissible ordinals, Ph. D. Thesis, Massachusetts Institute of Technology, 1976.

3. M. Lerman, On suborderings of the $\alpha$-recursively enumerable $\alpha$-degrees, Ann. Math. Logic 4 (1972), 415-443. MR 48 \#5835.

4. W. Maass, Inadmissibility, tamely RE sets and the admissible collapse, Ann. Math. Logic (to appear).

5. G. E. Sacks and S. G. Simpson, The $\alpha$-finite injury method, Ann. Math. Logic 4 (1972), 343-368. MR 51 \#5277.

6. R. Shore, The recursively enumerable $\alpha$-degrees are dense, Ann. Math. Logic 9 (1976), 123-155.

\section{DEPARTMENT OF MATHEMATICS, UNIVERSITY OF CHICAGO, CHICAGO}

ILLINOIS 60637

DEPARTMENT OF MATHEMATICS, HARVARD UNIVERSITY, CAMBRIDGE, MASSACHUSETTS 02138

DEPARTMENT OF MATHEMATICS, MASSACHUSETTS INSTITUTE OF TECHNOLOGY, CAMBRIDGE, MASSACHUSETTS 02139 